\title{
Publisher Correction: Geoscience on the chopping block
}

Correction to: Nature Reviews Earth \& Environment https://doi.org/10.1038/s43017-021-00216-1 published online 8 September 2021.

The sentence originally beginning 'In Australia, Macquarie University and the University of Newcastle axed their entire Earth science departments, ...' has been modified to 'In Australia, Macquarie University axed its entire Earth science department, ...'. The HTML and PDF versions have been corrected.

https://doi.org/10.1038/s43017-021-00227-y I Published online 23 September 2021

๑) Springer Nature Limited 2021 